\title{
Abstracts from Nippon Eiseigaku Zasshi (Japanese Journal of Hygiene) vol. 70, no. 1
}

(C) The Japanese Society for Hygiene 2015

\section{WHO Framework Convention on Tobacco Control (FCTC) Article 8: protection from exposure to tobacco smoke}

Nippon Eiseigaku Zasshi, 70, 3-14 (2015)

Hiroshi Yamato, Ying Jiang, Masanori Ohta

Department of Health Development, Institute of Industrial Ecological Sciences, University of Occupational and Environmental Health, Japan

It is necessary to implement $100 \%$ smoke-free environments in all indoor workplaces and indoor public places in order to protect people from exposure to second-hand tobacco smoke (SHS). Forty-four countries have already implemented comprehensive smoke-free legislations according to the Framework Convention on Tobacco Control (FCTC) Guidelines on protection from exposure to tobacco smoke. The Occupational Safety and Health Law (OSHL) was partially revised to strengthen the countermeasures against SHS in Japan in 2014. However, the revision was only minimal. Firstly, it is necessary to make efforts to implement countermeasures against SHS (their implementations are not obligatory, as required in Article 8). Secondly, the revised OSHL allowed the implementation of designated smoking rooms inside workplaces (Article 8 requires $100 \%$ smokefree environments). Thirdly, revised OSHL does not effectively cover the small-scale entertainment industry so that workers in restaurants and pubs will not be protected from occupational SHS. We explain the importance of implementation of $100 \%$ smoke-free environments by law, using the data on leakage of smoke from designated smoking rooms, and occupational exposure to SHS among service industry workers. The decrease in the incidence of smoking-related diseases in people where a comprehensive smoke-free law is implemented is also introduced. These data and information should be widely disseminated to policy makers, media, owners of service industries, and Japanese people.

The need for tobacco control in Japan based on Articles 9 and 10 of the World Health Organization Framework Convention on Tobacco Control (WHO FCTC), regulation of the contents of tobacco products, and regulation of tobacco product disclosures

Nippon Eiseigaku Zasshi, 70, 15-23 (2015)

Yohei Inaba, Shigehisa Uchiyama, Naoki Kunugita

Department of Environmental Health, National Institute of Public Health

In Japan, ultralow-nicotine cigarette brands with ventilation holes on the cigarette filters have been widely marketed to smokers. The use of these cigarette brands leads to compensation smoking. Menthol cigarette brands that have a cooling and numbing effect are also sold. In 2013, smokeless tobacco products similar to the Swedish snus, which is banned for sale in the European Union except in Sweden, were released in Japanese markets. These tobacco products have "toxicity," have a "ventilation filter," are "attractive," and promote "dependence." Tobacco smoke and smokeless tobacco are classified into "Group 1: Carcinogenic to humans" by the International Agency for Research on Cancer (IARC). The purpose of Articles 9 and 10 of the World Health Organization Framework Convention on Tobacco Control (WHO FCTC) is to regulate the contents of tobacco products as well as to regulate tobacco product disclosures. The implementation of some other articles has gradually advanced in Japan. However, that of Articles 9 and 10 is late. Japanese governmental authorities are being urged to immediately implement Articles 9 and 10 . 


\section{WHO Framework Convention on Tobacco Control (FCTC) Article 11: packaging and labelling of tobacco products}

Nippon Eiseigaku Zasshi, 70, 24-32 (2015)

Kanae Bekki, Yohei Inaba, Naoki Kunugita

Department of Environmental Health, National Institute of Public Health

The World Health Organization (WHO) Framework Convention on Tobacco Control (FCTC) requires member countries to implement measures aimed at reducing the demand for tobacco products. FCTC Article 11 describes the important forms of health communication and packaging regulations. And this article recommends on large pictorial health warnings and encourages more effective forms of disclosure on constituents and emissions. Furthermore, Article 11 recognizes the importance of the package as a promotional vehicle for tobacco companies and requires the removal of potentially misleading packaging information, including the terms "light" and "mild." The Conference of the Parties (COP) adopted guidelines for implementation of Article 11 on "Packaging and labelling of Tobacco Products". Some countries, such as Canada, the U.S.A., Australia, EU countries etc., positively promoted tobacco control by implementing counter-measures such as the graphic health warning labels and plain packages. These countermeasures showed the significant effects of decreasing smoking rate and preventing smoking initiation in young people. Furthermore, these warning labels were effective for the literally challenged. However, the Japanese government has not implemented these countermeasures, and only limited texts are shown on Japanese tobacco packaging. Therefore, Japan should emulate approaches taken by other countries, and promote the tobacco control policy in accordance with FCTC.

\section{Study on empowerment factors for career continuity of hospital nurses}

Nippon Eiseigaku Zasshi, 70, 33-39 (2015)

Hirosato Kato ${ }^{1}$, Yukie Ito ${ }^{1}$, Aki Yoshida ${ }^{1}$, Shizue Mizuno ${ }^{2}$, Kumiko Ogoshi $^{3}$, Tomoaki Imamura ${ }^{1}$

${ }^{1}$ Department of Public Health, Health Management and Policy, Nara Medical University

${ }^{2}$ Department of Nursing, Kyoto Koka Women's University

${ }^{3}$ Support Center for Female Researchers, Nara Medical University

Objectives: The resignation rate of nurses working in hospitals is extremely high. This study aims to identify both the factors related to the resignation of nurses and the empowerment factors supporting their job retention.

Methods: In 2011 we conducted a Web-based questionnaire survey of nurses with less than 10 years of working experience. There were 25 survey items including the following reasons for choosing the current employment position, intention of continuing work, sense of fulfillment and the presence or absence of problems in the current workplace. In addition, nurses who previously had resigned were asked why they left their last workplace.

Results: The reasons why they chose the current workplace were markedly different between the not-resigned (A group) and previously resigned (B group) nurses. As a result of cluster analysis, the reasons for resignation were classified into six clusters; "overwork", "burnout", "bad atmosphere", "work life balance factors", "working conditions" and "marriage".
Conclusions: In this study, it is shown that there is a positive correlation between the sense of fulfillment at work and the intention to do research work. It is suggested that encouragement of nurses to undertake research work is one of the empowerment factors supporting their job retention.

\section{Examination of diagnosis procedure combination survey data that influence function evaluation coefficient II}

Nippon Eiseigaku Zasshi, 70, 40-53 (2015)

Hisato Nakajima ${ }^{1}$, Kouya Yano ${ }^{2}$, Kaoko Nagasawa ${ }^{1}$, Eiji Kobayashi ${ }^{1}$, Kuninobu Yokota ${ }^{1}$

${ }^{1}$ Department of Medical Insurance Guidance Room, The Jikei University Hospital

${ }^{2}$ Department of Industrial Engineering and Management, College of Industrial Technology, Nihon University

Objectives: On the basis of diagnosis procedure combination (DPC) survey data, the factors that increase the value of function evaluation coefficient II were considered.

Methods: A total of 1,505 hospitals were divided into groups I, II, and III, and the following items were considered. (1) Significant differences in function evaluation coefficient II and DPC survey data. (2) Examination of using the Mahalanobis-Taguchi (MT) method. (3) Correlation between function evaluation coefficient II and each DPC survey data item.

Results: (1) Function evaluation coefficient II was highest in group II. Group I hospitals showed the highest bed capacity, and numbers of hospitalization days, operations, chemotherapies, radiotherapies and general anesthesia procedures. (2) Using the MT method, we found that the number of ambulance conveyances was effective factor in group I hospitals, the number of general anesthesia procedures was effective factor in group II hospitals, and the bed capacity was effective factor in group III hospitals. (3) In group I hospitals, function evaluation coefficient II significantly correlated to the numbers of ambulance conveyances and chemotherapies. In group II hospitals, function evaluation coefficient II significantly correlated to bed capacity, the numbers of ambulance conveyances, hospitalization days, operations, general anesthesia procedures, and mean hospitalization days. In group III hospitals, function evaluation coefficient II significantly correlated to all items.

Conclusions: The factors that improve the value of function evaluation coefficient II were the increases in the numbers of ambulance conveyances, chemotherapies and radiotherapies in group I hospitals, increases in the numbers of hospitalization days, operations, ambulance conveyances and general anesthesia procedures in group II hospitals, and increases in the numbers of hospitalization days, operations and ambulance conveyances. These results indicate that the profit of a hospital will increase, which will lead to medical services of good quality.

\section{Prevalence of eating disorders assessed using eating attitudes test-26 and their relevant factors in Japanese working women}

Nippon Eiseigaku Zasshi, 70, 54-61 (2015)

Miho Uehara $^{1}$, Hisataka Sakakibara ${ }^{2}$

${ }^{1}$ Former Nagoya University, Graduate School of Medicine Master Course

${ }^{2}$ Nagoya University, Graduate School of Medicine 
Objective: The purpose of the present study was to investigate the prevalence of eating disorders and their relevant factors in Japanese women.

Methods: Anonymous self-administered questionnaire surveys of 3023 working women aged 20-39 were conducted during health checkups in Tokyo, Nagoya, Osaka, and Fukuoka in 2012. Eating disorders were assessed using eating attitudes test-26 (EAT-26). The factors related to EAT-26 scores $\geq 20$ were analyzed by multiple logistic regression.

Results: The prevalences of eating disorders among the participants with EAT-26 scores $\geq 20$ were $2.4 \%$ [95\% confidence interval (95\% CI): $1.8-2.9 \%$ ] of all the participating women aged 20-39, $3.4 \%$ (95\% CI: $2.5-4.3 \%)$ of women in their $20 \mathrm{~s}$, and $1.2 \%(95 \%$ CI: $0.6-1.8 \%)$ of women in their $30 \mathrm{~s}$. Logistic regression analysis showed that EAT-26 scores of $\geq 20$ were associated with the following variables: perceived ideal BMI $\leq 17.5 \mathrm{~kg} / \mathrm{m}^{2}$ (OR: $4.55,95 \%$ CI: 2.08-9.93), change in body weight in the previous 6 months (OR: 2.83, $95 \%$ CI: $1.61-4.95$ ), being 20-29 years of age (OR: 2.64, $95 \%$ CI: $1.42-4.89)$, and the perception of being fat (OR $=2.54,95 \% \mathrm{CI}$ : 1.24-5.18).

Conclusions: The prevalence of eating disorders with EAT-26 scores $\geq 20$ was $2.4 \%$ (95\% CI: $1.8-2.9 \%$ ) among Japanese working women aged $20-39$. EAT- 26 scores $\geq 20$ were most closely associated with a perceived ideal BMI of $\leq 17.5 \mathrm{~kg} / \mathrm{m}^{2}$. Eating disorders may be more prevalent among women who want to lose weight to achieve a BMI of $\leq 17.5 \mathrm{~kg} / \mathrm{m}^{2}$, and these women should be carefully monitored.

\section{Prediction of upper limb recovery in the acute phase of cerebrovascular disease-study design and socio- demographic profiles, medical profiles, and acute symptoms of participants at baseline}

Nippon Eiseigaku Zasshi, 70, 62-68 (2015)

Toshimasa Sone $^{1}$, Naoki Nakaya ${ }^{2}$, Kazuaki Iokawa ${ }^{1}$, Keiichi

Hasegawa $^{3}$, Tetsu Tsukada ${ }^{3}$, Mariko Kaneda ${ }^{3}$, Toyohiro Hamaguchi ${ }^{4}$

${ }^{1}$ Course of Occupational Therapy, Department of Rehabilitation, Faculty of Health Science, Tohoku Fukushi University

${ }^{2}$ Department of Preventive Medicine and Epidemiology, Tohoku Medical Megabank Organization, Tohoku University

${ }^{3}$ Department of Rehabilitation, Takeda General Hospital

${ }^{4}$ Department of Occupational Therapy, School of Health and Social Services, Saitama Prefectural University

Objectives: In this study, we aim to predict the recovery of upper limb function in patients in the acute phase of cerebrovascular disease. Here, we describe the study design and patients' characteristics at baseline.

Methods: Subjects were admitted to Takeda General Hospital with cerebrovascular disease, and were prescribed to undergo rehabilitation. The assessment was conducted between June 2010 and March 2011. The baseline survey assessed the following factors: socio-demographic profiles (age, sex, number of family members, key person, occupation, and activities in daily life), medical profiles (types of cerebrovascular disease, location of brain damage, and history of diseases), and acute symptoms (Japan Coma Scale score, the Barre arm sign, the Brunnstrom recovery stage, tendon reflex, sensation, pain, ataxia, range of motion, muscle tone, grip strength, agnosia, apraxia, aphasia, dysarthria, the Hasegawa Dementia Scale-Revised score, and the Barthel index).

Results: A total of 357 patients with cerebrovascular disease were analyzed on the basis of diagnosis [cerebral infarction (CI; $77.6 \%$ ), cerebral hemorrhage $(\mathrm{CH} ; 20.2 \%)$, subarachnoid hemorrhage (SAH; $2.2 \%)$ ]. There was a significant difference in the average age between the types of cerebrovascular disease. In acute symptoms, sensation, grip strength, cognitive function, and activities of daily living were statistically significantly different between the types of cerebrovascular disease.

Conclusions: In this report, we described the study design and characteristics of patients with cerebrovascular disease at baseline. In the future, this study could be used for predicting the recovery of upper limb function in these patients.

\section{Comprehensive study on the prevention of food poisoning through the investigation of an affected hospital food service facility}

Nippon Eiseigaku Zasshi, 70, 69-80 (2015)

Satoshi Kubota ${ }^{1}$, Hiromi Kawai ${ }^{2}$

${ }^{1}$ Kuroshio Science Unit, Multidisciplinary Cluster, Kochi University

${ }^{2}$ Department of Food and Nutrition, Faculty of Contemporary Human Life Science, Tezukayama University

Objectives: In Japan, more than 20,000 people suffer from various types of food poisoning annually. In this paper, we discuss the prevention of food poisoning in hospital food service facilities from the perspective of hygiene management and organizational behavior.

Methods: We inspected the kitchen environment and the meal preparation process in a hospital food service facility in Japan that had been the site of a food poisoning incident. To clarify the present state of hygiene management, interviews were conducted with both the head of the nutrition and food service section and the administrative manager. In addition, questionnaires were distributed to the food service staff to assess their level of satisfaction with the working environment.

Results: The facility had been built about 10 years previously and was well maintained. Meal preparations were performed according to the operation manual, and education and training for the food service staff were carried out daily. No problems were evident regarding hygiene management. However, concerning organizational behavior, the satisfaction level of the staff was found to be relatively low, which may have led to a reduction in their organizational commitment and a decrease in their performance.

Conclusions: To aid in the prevention of food poisoning incidents in hospital food service facilities, it is essential not only to conduct standard hygiene management and training, but also to consider the organizational behavior of the food service staff.

Study on health support strategies by analyzing the diet, alcohol intake, and smoking behavior of university students: examination of non-communicable disease risk factors according to their sex, age and living arrangement

Nippon Eiseigaku Zasshi, 70, 81-94 (2015)

Junichi Kasamaki

Institute of Humanities, Social Sciences and Education, Niigata University

Objectives: The purpose of this study was to understand the diet, drinking, and smoking behaviors of university students and to analyze 
the health behaviors that could be a risk factor for non-communicable diseases (NCDs) in order to contribute to the promotion of NCD prevention in youth.

Methods: The survey was carried out using a questionnaire with closed questions including items about health behaviors. The subjects surveyed were students of 10 universities on the main island of Japan (1,196 valid responders).

Results: The score for the nutritional balance was significantly low in the group living alone for both students in their teens and $20 \mathrm{~s}$. For the frequency of not eating breakfast, results suggest that living alone and increase in age are related to the lack of breakfast for both males and females. Teenage males living alone tended to lack in consideration for the intake of animal fat than those not living alone. The females showed a higher tendency to eat sweets and snacks during the day than the males. For the males who living alone, results suggest that they tended to have a higher or equal alcohol intake to females in their $20 \mathrm{~s}$ and males in their $20 \mathrm{~s}$ not living alone even when they are underage. Males in their $20 \mathrm{~s}$ tended to have a higher amount and frequency of smoking than other groups regardless of their living arrangement.

Conclusions: Accumulation of health behavior that could be a risk for NCDs was found in some of the groups, such as males living alone. 